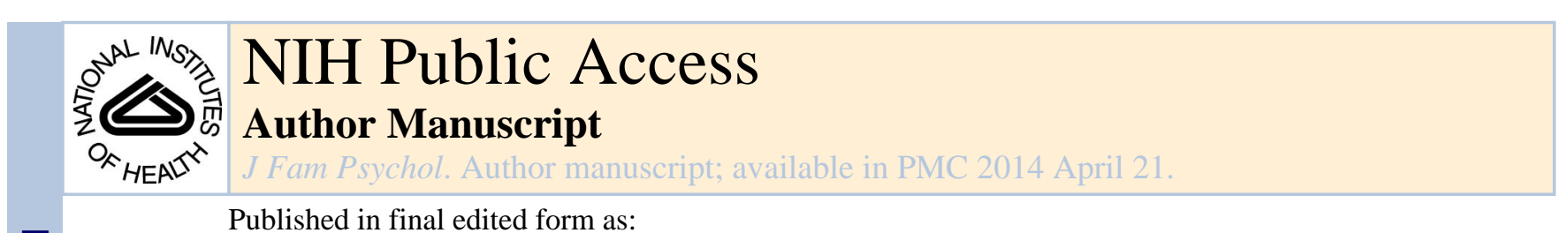

Published in final edited form as:

J Fam Psychol. 2012 June ; 26(3): 453-463. doi:10.1037/a0028332.

\title{
Perceived Partner Support in Pregnancy Predicts Lower Maternal and Infant Distress
}

\author{
Lynlee R. Tanner Stapleton, \\ University of California, Los Angeles \\ Christine Dunkel Schetter, \\ University of California, Los Angeles \\ Erika Westling, \\ Oregon Research Institute, Eugene, Oregon \\ Christine Rini, \\ University of North Carolina, Chapel Hill
}

Laura M. Glynn,

Chapman University and University of California, Irvine

Calvin J. Hobel, and

University of California, Los Angeles, and Cedars-Sinai Medical Center, Los Angeles, California

Curt A. Sandman

University of California, Irvine

\section{Abstract}

Maternal postpartum emotional distress is quite common and can pose significant risk to mothers and infants. The current study investigated mothers' relationships with their partners during pregnancy and tested the hypotheses that perception of prenatal partner support is a significant predictor of changes in maternal emotional distress from midpregnancy to postpartum, and contributes to maternal ratings of infant distress to novelty. Using a prospective longitudinal design, 272 adult pregnant women were interviewed regarding their partner support, relationship satisfaction, and interpersonal security (attachment style and willingness to seek out support), and they completed standardized measures of prenatal symptoms of depression and anxiety (distress). At 6 to 8 weeks' postpartum, mothers reported these symptoms again and completed measures of their infants' temperament. Structural equation modeling (SEM) was used to test direct and indirect contributions of partner support, relationship satisfaction, and interpersonal security to maternal and infant postpartum distress. Mothers who perceived stronger social support from their

\section{(C) 2012 American Psychological Association}

Correspondence concerning this article should be addressed to Christine Dunkel Schetter, Department of Psychology, University of California, Los Angeles, 1285 Franz Hall, Box 951563, Los Angeles, CA 90095-1563. dunkel@psych.ucla.edu.

Lynlee R. Tanner Stapleton and Christine Dunkel Schetter, Department of Psychology, University of California, Los Angeles; Erika Westling, Oregon Research Institute, Eugene, Oregon; Christine Rini, Department of Health Behavior, University of North Carolina, Chapel Hill; Laura M. Glynn, Department of Psychology, Chapman University, and Department of Psychiatry \& Human Behavior, University of California, Irvine; Calvin J. Hobel, Department of Obstetrics \& Gynecology, University of California, Los Angeles, and Cedars-Sinai Medical Center, Los Angeles, California; Curt A. Sandman, Department of Psychiatry \& Human Behavior, University of California, Irvine. 
partners midpregnancy had lower emotional distress postpartum after controlling for their distress in early pregnancy, and their infants were reported to be less distressed in response to novelty. Partner support mediated the effects of mothers' interpersonal security and relationship satisfaction on maternal and infant outcomes. A high-quality, supportive partner relationship during pregnancy may contribute to improved maternal and infant well-being postpartum, indicating a potential role for partner relationships in mental health interventions, with possible benefits for infants as well.

\section{Keywords}

social support; partner relationship; postpartum mental health; pregnancy; infant temperament

Maternal emotional distress during pregnancy and after birth represents a potential risk to the well-being of mothers and their families. Approximately $25 \%$ of women experience elevated symptoms of depression postpartum (O'Hara \& Swain, 1996), and rates may reach $40 \%$ to $50 \%$ in ethnic and racial minority populations (Yonkers et al., 2001). Anxiety is also quite prevalent during pregnancy and postpartum, with $5 \%$ to $8 \%$ of mothers estimated to meet diagnostic criteria for an anxiety disorder (Ross \& McLean, 2006). Depression and anxiety are often comorbid, and even symptoms that do not meet diagnostic thresholds can contribute to adverse birth outcomes, poorer postnatal well-being, and significant functional impairment for mothers (Dunkel Schetter, 2011; Field et al., 2003; Gotlib, Lewinsohn, \& Seeley, 1995).

Maternal emotional distress (mainly depressive and anxious symptoms) has biological concomitants with known adverse effects for neonatal and infant development. Elevations in prenatal maternal stress hormones predispose the fetus to be more sensitive and reactive to stress (Davis, Glynn, Waffarn \& Sandman, 2010; O’Donnell, O'Connor, \& Glover, 2009). Maternal distress and stress-related neuroendocrine changes in mid-to-late pregnancy have been linked to more difficult, fussy, and fearful early temperament and higher reactivity to novelty, as rated by independent observers and maternal reports (Davis et al., 2007; 2010; Martin, Noyes, Wisenbaker, \& Huttunen, 1999), as well as longer-term developmental effects such as cognitive, emotional, and behavioral disorders (e.g., Van den Bergh, Mulder, Mennes, \& Glover, 2005). Postpartum symptoms of emotional distress have also been associated with more negative maternal attitudes about one's infant, reduced parenting sensitivity, and increased intrusiveness and withdrawal which in turn predict infant fear dysregulation and stress reactivity (Feldman et al., 2009). Therefore, it is imperative to search for protective factors associated with lower maternal postpartum distress in order to contribute to improvements in maternal and child health.

Social support is commonly studied as a predictor of better mental and physical health; during pregnancy and postpartum, support (defined as interpersonal exchanges designed to meet the needs of another, or perceived availability of such resources; Dunkel Schetter \& Brooks, 2009) has been moderately to strongly associated with lower maternal postpartum depression and anxiety (Beck, 2001; Robertson, Grace, Wallington, \& Stewart, 2004). Furthermore, research shows that enacted or received support from a partner surrounding 
pregnancy predicts maternal prenatal and postpartum mental health (Dennis \& Ross, 2006; Rini, Dunkel Schetter, Hobel, Glynn, \& Sandman, 2006). When other support sources are absent or inadequate, intensive support from health care professionals can also improve maternal and child outcomes in at-risk families (Olds et al., 1997).

Relationship science provides insight into ways in which partner support may benefit perinatal well-being in conjunction with related constructs such as general satisfaction with the relationship and one's comfort and willingness to seek and receive support from others. The Relationship Enhancement Model (Cutrona, Russell, \& Gardner, 2005), for example, posits that a partner's provision of consistent and effective support behavior can increase perceptions of the partner as dependable and trustworthy as well as supportive, which in turn enhances relationship satisfaction and a person's psychological well-being and physical health. Consistent with these premises, one longitudinal study of pregnancy found that a multidimensional latent construct of effective prenatal support from the baby's father was associated with relationship satisfaction and interpersonal orientation. The effective support latent construct also predicted decreases in prenatal anxiety across pregnancy (Rini et al., 2006). However, this study did not examine depressive symptoms and stopped short of examining postpartum outcomes.

A key premise of support-based prenatal interventions has been that support is a central predictor of outcomes, without ever considering other aspects of close relationship functioning. However, supportive interactions occur within the broader context of a relationship and its history (Frazier, Tix, \& Barnett, 2003), and expectations and attributions regarding the relationship influence perceptions of discrete partner behaviors (Bradbury \& Fincham, 1990). Relationship satisfaction, or global evaluations of the relationship across multiple domains (Bradbury, Fincham, \& Beach, 2000), influence both support behaviors and perceptions of support (Pasch \& Bradbury, 1998). Relationship satisfaction and related aspects of relationship quality such as intimacy are themselves robust predictors of postpartum mental health as well (Beck, 2001; Robertson et al., 2004; Whisman, Davila \& Goodman, 2011).

Close personal relationships and the support that they offer are further influenced by a person's general expectations about and sense of security in close relationships which are rooted in early experience. Adult attachment theory posits that individuals who feel more secure in close interpersonal relationships will have more positive and satisfying romantic relationships compared with those who experience anxiety about rejection or avoid closeness (e.g., Hazan \& Shaver, 1987). Research confirms that individuals with more secure attachment styles and better integration into their social networks report higher quality relationships, more effective support from their partners, and more positive expectations about partner support (Cobb, Davila, \& Bradbury, 2001; Davila \& Kashy, 2009; Rini et al., 2006). Therefore, secure adult attachment style and positive orientation toward seeking help from others, conceptualized together here as "interpersonal security," should be examined as part of the context within which support in a specific couple relationship takes place. In the current study, we focus on relationship satisfaction and interpersonal security as general factors associated with, though clearly distinct from, more 
specific perceptions of partner support in order to begin to clarify the role of partner support in predicting postpartum mental health.

We also sought to expand upon existing research by exploring the associations between partner relationship factors and infant temperament. Partner support during pregnancy has not yet been directly examined as a predictor of maternal reports of infant temperament, but related evidence supports the plausibility of this link. Stressful partner relationship experiences during pregnancy, many of which reflect low support, have been associated with greater observed infant fear (Bergman, Sarkar, O'Connor, Modi, \& Glover, 2007), and married parents' positive and negative perceptions of their relationship have predicted changes in infants' negative affect over time (Belsky, Fish, \& Isabella, 1991). A prenatal coparenting intervention that promoted effective coordination and support between parents found positive effects on increased infant soothability and attention (Feinberg \& Kan, 2008), and it is plausible that supportive partners engage in more sensitive parenting behavior, which has been associated with lower infant stress reactivity (Feldman et al., 2009). Finally, partner support in the first month postpartum has been associated with infant stress reactivity and affective responsiveness during subsequent mother-child interactions (Crnic, Greenberg, Ragozin, Robinson, \& Basham, 1983). We therefore hypothesized that partner support might be associated with infant temperamental reactivity both via associations with lower maternal emotional distress during pregnancy and postpartum and via direct pathways. Gaining a better understanding of the role of partner support in shaping maternal and infant distress is critical to building theory and potential interventions.

\section{Study Overview and Hypotheses}

Consistent with previous longitudinal, developmental studies that take a family systems approach to the transition to parenthood (e.g., Belsky et al., 1991; Houts, Barnett-Walker, Paley, \& Cox, 2008), this study examined maternal perceptions of partner support and related relationship factors (partner relationship satisfaction and maternal interpersonal security) as predictors of maternal postpartum emotional distress (anxiety and depression) and infant temperament. Specifically, we sought to understand more clearly how partner support is related to these maternal and infant outcomes after accounting for more general relationship functioning and attachment-related expectations about close relationships. We used a large, longitudinal dataset with a sociodemographically diverse sample and combined the study of partner support, relationship satisfaction, and interpersonal security in the prediction of pre- and postpartum maternal emotional distress and infant temperament all within one study. Using structural equation modeling, we provide new evidence of unique associations between partner support and changes in maternal depressive and anxiety symptoms from pregnancy to postpartum, as well as maternal reports of a key aspect of infant temperament (distress to novelty).

A model of the hypothesized pathways is presented in Figure 1. The study hypotheses were that: (1) maternal interpersonal security and relationship satisfaction would covary inversely with prenatal maternal emotional distress and positively influence perceptions of partner support, consistent with prior research (Rini et al., 2006); (2) higher ratings of prenatal partner support would predict lower maternal postpartum emotional distress, as indicated by 
symptoms of depression and anxiety when controlling for prenatal symptoms; (3) partner support would mediate associations of relationship satisfaction and interpersonal security with postpartum outcomes; and (4) prenatal and postpartum maternal emotional distress would be associated with more distressed infant temperament. We also tested a set of hypotheses regarding indirect effects of support via reductions in maternal emotional distress based on past research (Davis et al., 2007) and direct, inverse effects of partner support on infant temperament.

\section{Method}

\section{Study Design and Procedure}

The current study used a subsample of participants from the Multi-Site Behavior in Pregnancy Study (MS-BIPS), a prospective study of psychosocial factors during pregnancy. Data was collected from women at prenatal clinics affiliated with two large urban medical centers that both serve diverse populations in terms of maternal education, income and ethnicity/race. The majority in one site is Latina (61\% Latina, 25\% White and 6\% Asian), and the other site is more heterogeneous (40\% Caucasian, 31\% Hispanic, 29\% African American). The study sample was recruited and followed by research nurses, physicians, and research staff with data collected at three intervals over the course of pregnancy (T1: 18 -20 weeks' gestation; T2: 24 -26 weeks' gestation; T3: 28 -32 weeks' gestation) and at 6 8 weeks' postpartum (P1). The T1 assessment was timed so as to reduce sampling bias because women of lower socioeconomic status or with unplanned pregnancies begin prenatal care later. Semistructured interviews and questionnaires were administered by trained interviewers in clinic settings within private rooms or areas. All measures were designed for a sixth-grade educational level and piloted extensively.

\section{Participants}

Eligibility-Participants were pregnant women at least 18 years of age who were at 18 weeks' singleton gestation or less at enrollment and were able to be interviewed in English. The larger MS-BIPS project investigated physiological changes during pregnancy and excluded women using tobacco or controlled substances or who had medical conditions involving cardiovascular, neuroendocrine, hepatic or renal functioning. Enrollment was not restricted by mental health history, parity, ethnicity or other factors. A total of 1,189 pregnant women were approached during the study period, of whom $63 \%$ met eligibility criteria; $67 \%$ of those eligible were consented into the study $(n=499)$. The main reasons for ineligibility (in order of frequency) were non-English speaking, pregnancy was too far advanced, multiple gestation, and smoking. The protocol was approved by the institutional review boards at all institutions.

Current sample-As this study focused on partner relationships, only women who reported being in an intimate relationship with a partner at $\mathrm{T} 1$ were considered for inclusion $(95 \% ; n=475)$. Because there were missing data due to attrition at various assessment points, only cases that provided complete data on variables examined here were used $(n=$ 272). 
The majority of the current sample was married at T1 (79\%), and all but three reported their partner was the baby's father. Half of the sample self-identified as non-Hispanic White (53\%) with subgroups of Latina (21\%), African American (11\%), and Asian American $(10 \%)$. Mean maternal age was 30 years $(S D=5.1)$. Distribution of annual household income was $16 \%$ under $\$ 30,000,27 \%$ from $\$ 30,000-60,000,24 \%$ from $\$ 60,000-90,000$, and $33 \%$ over $\$ 90,000$ (mean household size $=2.9$ persons). Twelve percent had a high school diploma or less education; $36 \%$ held a technical degree, certificate, associate's degree, or attended some college; and 52\% held a bachelor's degree or higher. Over half were having a first birth (58\%). Infants were born on average at 39.0 weeks' gestation ( $S D=$ 1.7), and exactly half were male. Participants not included in the current sample because of attrition and missing data $(n=203)$ were significantly less likely to be married $(63 \%)$, to have a bachelor's degree (34\%), to be non-Hispanic White (41\%), and to be expecting a first child $(48 \%)$, and had lower mean household incomes $(\$ 50,000-60,000)$ compared with participants in the current sample. Participants with missing data did not differ from those with complete data $(p s>.10)$ in maternal age, partner support, postpartum depression, or infant distress but reported significantly lower relationship satisfaction, more prenatal and postpartum anxiety, and marginally more prenatal depression (when data were available).

\section{Measures}

Measures were selected on the basis of evidence of their reliability and validity in prior similar studies, with the exception of one new measure of pregnancy-specific support designed for this investigation (described below). Some were modified for length, ease of use, and comprehensibility as described. Cronbach's alpha coefficients for computed variables are presented in Table 1. All alpha coefficients were acceptable to good.

Maternal interpersonal security (T1) - Interpersonal security was measured with a combination of two standardized instruments reflecting adult attachment style and willingness to seek and accept help from others. Attachment style was assessed using a version of the Adult Attachment Scale (AAS; Collins \& Read, 1990) that contained three subscales (five items each): comfort with closeness (e.g., "I worry about people getting too close to me"), comfort depending on others (e.g., "I find it difficult to trust others completely"), and anxiety about being rejected by others (e.g., "I often worry that romantic partners won't want to stay with me"). Items were rated on a 5-point scale from $1=$ "not at all like $m e$ " to 5 = "very much like $m e$ " and averaged within subscale, with higher scores indicating more secure attachment style.

The Network Orientation Scale (NOS; Vaux, Burda, \& Stewart, 1986) assessed how open and willing participants generally were to seek and accept support from others (e.g., "It's okay to ask favors of people"). This measure has been used to study social relationships outside the pregnancy context and is associated moderately with adult attachment (e.g., Wallace \& Vaux, 1993). Responses to items were made on a scale from 1 (strongly disagree) to 4 (strongly agree). Based on factor analyses research (Rini et al., 2006), 13 of the original 20 items were scored in published into an index with higher scores indicating a more favorable network orientation. 
Relationship satisfaction (T1)—The Marital Adjustment Test (MAT; Locke \& Wallace, 1959) is a well-validated 15 -item measure of relationship quality measuring global partner relationship quality and satisfaction. Items concern the degree of happiness and agreement between partners on various issues and were reworded slightly to apply to both married and unmarried relationships. Items were weighted and summed as suggested by Freeston and Pléchaty (1997). An additional item, based on the MAT, asked participants to rate the amount of intimacy and emotional closeness in their relationship using a 7-point scale from $1=$ "almost no closeness or intimacy" to 7 = "a great deal of emotional closeness and intimacy."

Partner support (T2)—Prenatal support from the baby's father was assessed with a combination of two measures, one of support effectiveness and one of pregnancy-specific received support. The Social Support Effectiveness (SSE) interview was previously validated on the MS-BIPS sample (Rini et al., 2006) and further validated by Rini et al. (2011) in a different population. This measure asked women to rate partner support quantity and quality (e.g., "How good is the match between the amount of help he offers and the amount you need?"), how skillfully it was provided, and how accessible support was for each of three types of support: Task (5 items), Informational (3 items), and Emotional Support (5 items). Support negativity (e.g., makes you feel guilty, inadequate; lets you down) was assessed using six items, each rated on a 5-point rating scale ranging from $1=$ "always" to $5=$ "never." The four subscales were scored with high scores reflecting high support and low negativity per Rini et al. (2006).

No existing validated measure was identified to assess pregnancy-specific support needs, so a set of questions was created for this study based on prior research (Collins et al., 1993). Women were queried about any extra help they needed "because of the current pregnancy" in terms of everyday tasks and responsibilities, material things, emotional support (e.g., listening, understanding), and information/advice. Specific questions concerned how often the baby's father did each behavior, how often others did each, and how well each of their needs of each type were met. A composite score was created from four items that assessed frequency of pregnancy-related task and emotional support from the baby's father (each rated on a 5-point scale from $1=$ "never" to $5=$ "very often") and satisfaction with each type of support overall (rated on a 5-point scale from $1=$ "not at all satisfied" to $5=$ "completely satisfied"). Items were standardized then averaged, with higher scores indicating higher pregnancy-specific support quantity and quality. The 4 items were highly intercorrelated and formed the best index from the set of new items for testing pregnancy-specific partner support.

Maternal emotional distress (T1, P1)—Current emotional distress was measured preand postpartum with two validated scales for anxious and depressive symptoms. State anxiety was assessed with the state subscale of the State-Trait Anxiety Inventory (STAI; Spielberger, 1983). It consists of a series of adjectives rated on a four-point scale ( 1 = "not at all" to $4=$ "very much" as to how often the person experienced each (e.g., worried, calm, jittery). Responses were averaged across all items. During pregnancy the short 10-item version was used, whereas the longer 20-item version was administered during postpartum. 
Depressive symptoms were measured with the short version of the Center for Epidemiological Studies Depression Scale (CES-D; Santor \& Coyne, 1997). This measure has been used extensively with nonclinical samples and published studies demonstrate validity and internal consistency. Nine items were each rated on a 4-point Likert scale with a range of 0 to 3 assessing the frequency in days per week of different depressive symptoms (from "less than one day" to "5-7 days"). Two items that corresponded to physical symptoms of pregnancy or the postpartum period (i.e., "I felt that everything I did was an effort" and "My sleep was restless") were excluded from these analyses to provide a conservative, unconfounded measure of depressive mood (cf. Matthey \& Ross-Hamid, 2011). The remaining 7 items were averaged.

Infant distress to novelty (P1)-One aspect of infant temperament especially relevant to stress reactivity - infant distress to novelty—-was assessed using a modified version of the Infant Behavior Questionnaire (IBQ), a standardized instrument designed to assess infant temperament by maternal report (Rothbart, 1981). The IBQ was designed to minimize the influence of maternal bias by asking about specific infant behaviors. It has been found to have strong psychometric properties and is closely related to independent laboratory ratings of infant behavior (Gartstein \& Rothbart, 2003). It has the advantage that mothers observe their infants in many contexts which laboratory studies cannot. Because not all items were well suited to the young age of infants in this study, a subset of 20 age-appropriate items were chosen. Mothers rated their infants' behavior on each item on a 5-point Likert scale ranging from "never" to "always." Factor analysis of the 20 items revealed a primary 10item factor corresponding closely to the IBQ subscale for infant fear/distress to novelty (e.g., "How often in the past week did the baby fuss or cry when his or her face or hair was washed? $\ldots$ startle or cry at a sudden change in position?, ... show a negative response to a strange person?"). Scores on these items were summed to form an index of Infant Distress to Novelty.

\section{Results}

Descriptive statistics are presented in Table 1, including means and 95\% confidence intervals for observed variables. The current sample had a mean score on relationship satisfaction above the standard cutoff score of 100 which distinguishes distressed couples (low scores) from nondistressed couples (Abramowitz \& Sewell, 1980); overall 14\% of couples were in the clinically distressed range. Reports of depression and anxiety symptoms were low on average and intercorrelated as expected, sharing one third to half of their variance. Table 2 contains zero-order correlations among the primary study variables, which were consistent with hypotheses and provided a sound basis for model testing.

\section{Data Analytic Plan and Structural Equation Modeling}

First, we examined descriptive characteristics of the data and zero-order correlations between observed variables (using Bonferroni alpha correction) to provide initial confirmation of our theorized clusters of latent variables. Then, we used confirmatory factor analysis in EQS 6.1 (Bentler, 2005) to test further the fit of our observed measures to latent factors. Structural equation modeling (SEM) in EQS was then used to test the multiple 
hypothesized direct and indirect relationships between variables via maximum likelihood estimation with robust standard errors. This approach estimates all parameters simultaneously, controlling for all other effects in the model. The final model was compared to alternative models to test competing hypotheses.

Initial SEM analyses attempted to use full-information estimation methods (FIML) to include data from all participants who reported having a relationship at T1 $(n=475)$. However, participants who did not complete assessment at a given time point provided no data on primary variables of interest (e.g., partner support factor, postpartum functioning), and FIML models were underidentified and inestimable given the available data. Therefore, it was decided to use only cases that provided complete data.

Multiple indices assessed the fit of each model: the Satorra-Bentler $\chi^{2}$, which adjusts the typical $\chi^{2}$ for non-normally distributed data (S-B $\chi^{2}$; Bentler \& Dudgeon, 1996), the robust comparative fit index (RCFI; Bentler \& Dudgeon, 1996; Byrne, 1994), and the root-meansquare error of approximation (RMSEA; Hu \& Bentler, 1999). The $\chi^{2}$ tests the null hypothesis that the model perfectly fits the data structure but is commonly significant in samples greater than 200. The RCFI ranges from 0 to 1 , with values above .95 indicating good model fit. RMSEA values of less than .06 indicate good fit. (See Widaman \& Thompson, 2003, for an overview of fit indices.) Robust standard errors were used because of multivariate kurtosis in the data (Mardia's normalized estimates of 18.34 and 18.75 for the measurement and final model, respectively).

\section{Latent Variables and Confirmatory Factor Analysis}

Based on theory, previous research (Rini et al., 2006), correlations, and timing of administration, measures of interpersonal security, relationship satisfaction, partner support, and emotional distress were grouped to form five latent factors (Table 1). To prevent underidentification of the Relationship Satisfaction latent factor, we divided the MAT into two subsets by separating odd and even items (Little, Cunningham, Shahar, \& Widaman, 2002), creating a total of three satisfaction indicators (two MAT parcels plus the intimacy and closeness item). Similarly, the STAI and CES-D scales were each split into two arbitrary subsets at each time point to form a four-indicator maternal distress factor, in which residuals between parcels from the same measure were allowed to covary. The measurement model of five latent factors provided good fit to the data, S-B $\chi^{2}(156, N=$ $272)=271.32, p<.01, \mathrm{RCFI}=.97, \mathrm{RMSEA}=0.038$, with all proposed indicators loading between .50 and .98 on their respective factors at $p<.001$. Factor loadings remained highly similar in the final model.

\section{Hypothesized Structural Model}

The hypothesized predictive model (Figure 1) fit the data well: S-B $\chi^{2}(176, N=272)=$ $230.94, p=.003, \mathrm{RCFI}=.98$, RMSEA $=0.034$. However, the hypothesized direct path predicting infant distress to novelty from maternal prenatal emotional distress at $\mathrm{T} 1$ was not significant after controlling for postpartum emotional distress. The removal of this path resulted in a model with highly similar fit indices, S-B $\chi^{2}(177, N=272)=230.99, p=.004$, $\mathrm{RCFI}=.98, \mathrm{RMSEA}=0.034$, and the models were not significantly different according to 
the robust chi-square difference test (Satorra \& Bentler, 2001), $\Delta \chi_{\text {robust }}^{2}(\Delta d f)=0.009(1), p$ $=.92$. The more parsimonious final model is presented in Figure 2. All illustrated paths were significant in the hypothesized direction ( $p<.05$, two-tailed tests).

\section{Final Model}

The final predictive model as depicted in Figure 2 (all pathways significant at $p<.05$ ) replicated earlier findings that higher maternal interpersonal security and partner relationship satisfaction at $\mathrm{T} 1$ are correlated and positively associated with reports of stronger partner support. In addition, maternal interpersonal security and relationship satisfaction were inversely significantly related to maternal prenatal anxiety and depressive symptoms (T1 maternal emotional distress). Stronger perceived partner support during pregnancy predicted significantly lower postpartum maternal emotional distress $(\beta=-.15)$, controlling for prenatal levels, and partner support further predicted significantly lower infant distress to novelty $(\beta=-.21)$. Higher infant distress to novelty was also significantly and positively associated with maternal postpartum emotional distress $(\beta=.19)$. There were no significant direct effects between $\mathrm{T} 1$ and P1 variables other than continuity between maternal reports of emotional distress over time. Overall, the model fit the data very well and explained $39 \%$ of the variance in postpartum maternal emotional distress and $11 \%$ of the variance in infant distress to novelty.

Decomposition of estimated parameters into direct and indirect effects was also examined within EQS to assess the combined influence of $\mathrm{T} 1$ variables on postpartum maternal emotional and infant distress via intermediary variables. Interpersonal security, relationship satisfaction, and prenatal emotional distress each had significant indirect effects on infant distress $(\beta s=-.07,-.14$, and .10 , respectively, $p s<.05$ ) via prenatal partner support and postpartum maternal emotional distress, indicating mediation in the absence of direct effects. Interpersonal security did not have a significant indirect effect on postpartum emotional distress, and the indirect effect of relationship satisfaction was only marginally significant ( $\beta$ $=-.09, p<.10)$.

The final model combined depressive and anxiety symptoms into a latent maternal distress factor at T1 and at T2. However, we also examined a model that included the two affective variables (depressive and anxiety symptoms) as single, observed variables that were correlated. Findings were consistent with the final latent model, with two slight variations worth noting. First, partner support was more strongly associated with postpartum depressive symptoms than with anxiety, and second, maternal anxiety was directly associated with infant distress whereas depressive symptoms were not.

\section{Alternative Models}

To determine whether the model in Figure 2 represented the best fit to the data and to consider competing hypotheses, we tested three alternative models. A fully saturated model was tested allowing all paths among latent variables and infant distress to novelty to be freely estimated to ensure no important paths were omitted. No additional significant paths emerged. Next an alternative model that allowed direct predictive paths to be estimated between general relationship factors (i.e., interpersonal security and relationship 
satisfaction) and postpartum emotional distress, to test the possibility that maternal individual differences in interpersonal security and relationship satisfaction, rather than partner support, directly influence postpartum emotional distress. Another alternative model tested a direct effect of prenatal maternal emotional distress on ratings of partner support, to test the possibility that mothers' emotional state influenced her report of partner support beyond relationship satisfaction and interpersonal security. Evidence was inconsistent with each of these models. None of the additional paths were significant, and none of the alternative models significantly improved fit over the model in Figure 2. We also tested potential covariates (marital status, parity, ethnicity, income, problematic birth outcomes such as preterm delivery, and prenatal medical risk factors including hypertension and infections) individually as predictors of postpartum maternal emotional distress and of infant distress to novelty. None of the paths between covariates and these outcomes were significant, and their addition did not improve model fit.

\section{Discussion}

This study adds to our understanding of the importance of an expecting mother's relationship with her partner, especially as a provider of effective social support during pregnancy. The results indicated that the significant effects of interpersonal security and relationship satisfaction on maternal postpartum emotional distress were mediated by the perceived quantity and quality of partner support. Thus, our results are consistent with the conclusion that an expectant father's support is in part a function of the qualities of his relationship with the baby's mother and her attachment-based relationship expectations, but the perceived effectiveness of his support is a more proximal contributor to maternal postpartum emotional distress. These findings add to previous research which demonstrated that the quality of a couple's interactions predicts the parents' depression following childbirth (Houts et al., 2008). Of the various types of support measured here, emotional support loaded the highest on the partner support latent factor, suggesting that listening and affectionate behaviors might account for much of the observed associations between prenatal support and decreases in maternal emotional distress from pregnancy and postpartum.

This study's results also add to earlier findings showing that partner support predicted midpregnancy anxiety (Rini et al., 2006) by demonstrating associations between prenatal support and both depressive and anxious symptoms and by extending the timeframe both earlier in pregnancy and into postpartum. Furthermore, these findings were obtained in a more diverse sample than is typically seen in the transition to parenthood literature, and the final model showed similar fit to the data when covariates such as ethnicity, parity or pregnancy risks and complications were included. This is consistent with an earlier study, which found that ethnicity did not affect cross-sectional associations between overall support or closeness with partner and postpartum depression (Logsdon \& Usui, 2001). However, we note that attrition over time somewhat influenced the sociodemographic distribution of our sample, and findings may not generalize to very low-income or loweducated populations. 
Although this study is not experimental and cannot affirm causality, the results provide evidence that perceived partner support during pregnancy may contribute to less fearful or emotionally reactive infant temperament. One potential indirect mechanism is that the effective partner support and lower maternal emotional distress might both enable mothers to enact more sensitive and engaged parenting (Feldman et al., 2009), contributing to lower infant distress. Still, the direct effect of perceived prenatal partner support on lower reported infant distress to novelty suggests that a father's influence extends beyond his contribution to improved maternal emotional health. These results are newsworthy whether they reflect mothers' perceptions of her infant, her infant's actual behavior, or both, as is most likely the case.

Several possible mechanisms may be operating to explain the importance of the partner's behavior. Partner support during pregnancy may contribute to unmeasured mediators, such as better maternal health behavior (e.g., smoking) or improved prenatal care, and thereby indirectly influence infant outcomes via biological mechanisms including reduced maternal stress reactivity (Martin et al., 1999). It is also plausible that a partner's provision of support and involvement in the pregnancy is associated with other protective factors such as higher maternal personal resources (Dunkel Schetter, 2011; Dunkel Schetter \& Dolbier, 2011) or partner's involvement in or quality of parenting (Cox, Owen, Lewis, \& Henderson, 1989) which are associated with child temperament (Boyce et al., 2006). These and other possibilities may explain the direct effect of partner prenatal support on mothers' reports of later infant temperament. Future research exploring these potential mediators and other theoretically derived mechanisms is necessary to follow-up these findings. It should also be noted that, although the latent partner support factor was the only direct relationship-related predictor of maternal and infant outcomes, relationship satisfaction and interpersonal security were associated with support ratings and exerted indirect effects on infant distress. The current data support conceptual distinctions between these three factors consistent with theory, but indicate some combined influences such that mothers with higher quality partner relationships and more secure attachment styles are likely to have better mental health in pregnancy and postpartum. Therefore, support should generally be examined in the context of broader partner relationship functioning when considering potential family based interventions (Pietromonaco, Uchino, \& Dunkel Schetter, in press).

The current study did not find a direct association between maternal prenatal emotional distress and infant distress after controlling for postpartum maternal distress, which is inconsistent with an earlier report (Davis et al., 2007). However, the current study differed from the earlier one in important ways. First, we utilized structural equation modeling and included interpersonal relationship variables in the current model. When the relationship context was considered here, maternal prenatal distress exerted only an indirect influence on infant distress to novelty. In addition, Davis et al. assessed symptoms earlier in the second trimester (vs. here when assessed in mid to late pregnancy) and used a broader measure of infant temperament which included additional items on infant distress to limitations and fear. Thus, our results add to and complement the earlier findings and provide evidence that relationship factors might account for some of the association between a mother's prenatal distress and infant temperament. 
A few methodological and conceptual aspects of the current study are worth noting. Our study design involved multiple, detailed assessments of many constructs in a relatively large and somewhat diverse sample of pregnant women followed prospectively into the first few months postpartum. This avoided retrospective reporting biases and allowed examination of changes in maternal mental health over time. In addition, SEM modeling techniques permitted us to model latent constructs and to test the effects of prenatal partner support on postpartum outcomes while controlling for prenatal maternal emotional state and important relationship factors. Temporal ordering enhances the ability to infer causality; nonetheless, these results cannot firmly establish causality, and some associations may be bidirectional or involve third variables. Replication and randomized controlled trials of theory-based interventions designed to enhance partner support are needed to establish any causal effects on maternal and child outcomes.

Insomuch as these data were based on maternal self report, common method variance and other self-report biases may account for a portion of the observed associations. The infant distress variable, for example, may be argued to be, at least in part, a function of maternal affective symptoms (Pauli-Pott, Mertesacker, Bade, Haverkock, \& Beckmann, 2003). However, our final model continued to show significant direct associations between prenatal support and infant distress after controlling for the association between maternal postpartum emotional distress and infant distress. In addition, observed zero-order and final modeled associations between maternal emotional distress and ratings of infant temperament might reflect shared genetic variance or physiological programming effects of prenatal anxiety on the fetus, not merely measurement artifacts (Rothbart \& Hwang, 2002), an explanation worth exploring in future studies. While we cannot rule out the possibility that reliance on maternal reports are responsible for these results, or the possibility that some reciprocal influence of infant temperament on maternal adjustment exists, maternal perceptions are critical perspectives of her close relationships, of her experiences as a parent, and of her family functioning, and thus they have meaning and validity in and of themselves phenomenologically. Future research should include the perspectives of both partners regarding their supportive interactions to clarify further the role of partner support in postpartum maternal and infant adjustment. Other indicators of infant temperament should also be examined. In addition, attrition of participants of lower socioeconomic status and minority ethnicity over time limits generalizability to the entire population of pregnant women and emphasizes the need for additional efforts to enroll and retain these participants in future research.

The measures used to assess maternal depression and anxiety symptoms are validated screening tools but they are not diagnostic of major depressive disorder or anxiety disorders in mothers. On average, this sample reported mild to moderate symptoms of emotional distress. Nonetheless, the associations between prenatal support and postpartum outcomes were of moderate size. How these results would differ in a population of mothers with a wider range of mental health symptoms is unknown, but the effects are likely to be more pronounced in women with greater support needs. Tests of partner support in a sample of women with higher symptom levels or from a treatment-seeking population would be valuable in informing clinical assessment and intervention. 
In conclusion, results from this study are consistent with the argument that involvement of and support from the baby's father during pregnancy is associated with improved maternal mental health and may contribute to less distressed infant temperament. It is known that early infant distress behaviors, and associated heightened reactivity to stress, set the stage for later emotion regulation and mental health in children (Rothbart, Ahadi, \& Evans, 2000). Therefore, the current findings provide additional impetus for exploring prenatal partner support together with related aspects of the partner relationship as potential avenues to improve postpartum maternal mental health and child development. Past randomized controlled trials designed to increase the well-being of mothers and infants through social support intervention in pregnancy have had mostly no effects (Hodnett \& Fredericks, 2003). Exceptions (Norbeck, DeJoseph, \& Smith, 1996; Olds et al., 1997) have been closely guided by theoretical models and focused on specific types of relationships. Enhancing the partner's capacity to provide high quality social support during pregnancy, and potentially addressing underlying relationship and support expectations, may be more effective in reducing maternal postpartum anxiety and depression than attempts to increase general social support. At the same time, replication with objective measures of support and infant temperament and further investigation into the proposed mechanisms underlying associations between prenatal partner support and infant temperament are needed. This line of research has potential to produce more effective interventions to reduce the burden of perinatal maternal postpartum depression and anxiety and their contributions to child stress reactivity.

\section{Acknowledgments}

Support for this project was provided by the National Institute of Child Health and Human Development (HD28413) and the National Institute of Mental Health (MH15750). We thank Dr. Elysia Poggi Davis, members of Dr. Dunkel Schetter's UCLA lab (http://health.ucla.edu/CDS), and several anonymous reviewers for feedback on this article, and Dr. Anja Broda for her assistance with preliminary analyses.

\section{References}

Abramowitz SI, Sewell HH. Marital adjustment and sex therapy outcome. The Journal of Sex Research. 1980; 16:325-337.10.1080/00224498009551089

Beck CT. Predictors of postpartum depression: An update. Nursing Research. 2001; 50:275285.10.1097/00006199-200109000-00004 [PubMed: 11570712]

Belsky J, Fish M, Isabella RA. Continuity and discontinuity in infant negative and positive emotionality: Family antecedents and attachment consequences. Developmental Psychology. 1991; 27:421- 431.10.1037/0012-1649.27.3.421

Bentler, PM. EQS 6 Structural Equations program manual. Encino, CA: Multivariate Software; 2005. computer software

Bentler PM, Dudgeon P. Covariance structure analysis: Statistical practice, theory, and directions. Annual Review of Psychology. 1996; 47:563-592.10.1146/annurev.psych.47.1.563

Bergman K, Sarkar P, O’Connor TG, Modi N, Glover V. Maternal stress during pregnancy predicts cognitive ability and fearfulness in infancy. Journal of the American Academy of Child \& Adolescent Psychiatry. 2007; 46:1454-1463.10.1097/chi.0b013e31814a62f6 [PubMed: 18049295]

Boyce WT, Essex MJ, Alkon A, Goldsmith H, Kraemer HC, Kupfer DJ. Early father involvement moderates biobehavioral susceptibility to mental health problems in middle childhood. Journal of the American Academy of Child \& Adolescent Psychiatry. 2006; 45:1510-1520.10.1097/01.chi. 0000237706.50884.8b [PubMed: 17135997]

Bradbury TN, Fincham FD. Attributions in marriage: Review and critique. Psychological Bulletin. 1990; 107:3-33.10.1037/0033-2909.107.1.3 [PubMed: 2404292] 
Bradbury TN, Fincham FD, Beach SRH. Research on the nature and determinants of marital satisfaction: A decade in review. Journal of Marriage and the Family. 2000; 62:964-980.10.1111/j. 1741-3737.2000.00964.x

Byrne BM. Testing for the factorial validity, replication, and invariance of a measuring instrument: A paradigmatic application based on the Maslach Burnout Inventory. Multivariate Behavioral Research. 1994; 29:289-311.10.1207/s15327906mbr2903_5

Cobb RJ, Davila J, Bradbury TN. Attachment security and marital satisfaction: The role of positive perceptions and social support. Personality and Social Psychology Bulletin. 2001; 27:11311143.10.1177/0146167201279006

Collins NL, Dunkel Schetter C, Lobel M, Scrimshaw SC. Social support in pregnancy: Psychosocial correlates of birth outcomes and postpartum depression. Journal of Personality and Social Psychology. 1993; 65:1243-1243.10.1037/0022-3514.65.6.1243 [PubMed: 8295121]

Collins NL, Read SJ. Adult attachment, working models and relationship quality in dating couples. Journal of Personality and Social Psychology. 1990; 58:644- 663.10.1037/0022-3514.58.4.644 [PubMed: 14570079]

Cox MJ, Owen MT, Lewis JM, Henderson VK. Marriage, adult adjustment, and early parenting. Child Development. 1989; 60:1015-1024.10.2307/1130775 [PubMed: 2805879]

Crnic KA, Greenberg MT, Ragozin AS, Robinson NM, Basham RB. Effects of stress and social support on mothers and premature and full-term infants. Child Development. 1983; 54:209217.10.2307/1129878 [PubMed: 6831987]

Cutrona, CE.; Russell, DW.; Gardner, KA. The relationship enhancement model of social support. In: Kayser, K.; Bodemann, G.; Revenson, TA., editors. Couples Coping with Stress: Emerging Perspectives on Dyadic Coping. Washington, DC: APA; 2005. p. 73-95.

Davila J, Kashy DA. Secure base processes in couples: Daily associations between support experiences and attachment security. Journal of Family Psychology. 2009; 23:76- 88.10.1037/ a0014353 [PubMed: 19203162]

Davis EP, Glynn LM, Dunkel Schetter C, Hobel CJ, Chicz-DeMet A, Sandman CA. Prenatal exposure to maternal depression and cortisol influences infant temperament. Journal of the American Academy of Child \& Adolescent Psychiatry. 2007; 46:737-746.10.1097/chi.0b013e318047b775 [PubMed: 17513986]

Davis EP, Glynn LM, Waffarn F, Sandman CA. Prenatal maternal stress programs infant stress regulation. Journal of Child Psychology and Psychiatry. 2010; 52:119-129.10.1111/j. 1469-7610.2010.02314.x [PubMed: 20854366]

Dennis C, Ross L. Women's perceptions of partner support and conflict in the development of postpartum depressive symptoms. Journal of Advanced Nursing. 2006; 56:588-599.10.1111/j. 1365-2648.2006.04059.x [PubMed: 17118038]

Dunkel Schetter C. Psychological science on pregnancy: Stress processes, biopsychosocial models, and emerging research issues. Annual Review of Psychology. 2011; 62:531-558.10.1146/ annurev.psych.031809.130727

Dunkel Schetter, C.; Brooks, K. The nature of social support. In: Reis, HT.; Sprecher, S., editors. Encyclopedia of human relationships. Thousand Oaks, CA: Sage Publications; 2009. p. 1565-1570.

Dunkel Schetter C, Dolbier C. Resilience in the context of chronic stress in adults. Social and Personality Psychology Compass. 2011; 5:634- 652.10.1111/j.1751-9004.2011.00379.x

Feinberg ME, Kan ML. Establishing family foundations: Intervention effects on coparenting, parent/ infant well-being, and parent-child relations. Journal of Family Psychology. 2008; 22:253263.10.1037/0893-3200.22.2.253 [PubMed: 18410212]

Feldman R, Granat A, Pariente C, Kanety H, Kuint J, Gilboa-Schechtman E. Maternal depression and anxiety across the postpartum year and infant social engagement, fear regulation, and stress reactivity. Journal of the American Academy of Child \& Adolescent Psychiatry. 2009; 48:919927.10.1097/CHI.0b013e3181b21651 [PubMed: 19625979]

Field T, Diego M, Hernandez-Reif M, Schanberg S, Kuhn C, Yando R, Bendell D. Pregnancy anxiety and comorbid depression and anger: Effects on the fetus and neonate. Depression and Anxiety. 2003; 17:140-151.10.1002/da.10071 [PubMed: 12768648] 
Frazier PA, Tix AP, Barnett CL. The relational context of social support: Relationship satisfaction moderates the relations between enacted support and distress. Personality and Social Psychology Bulletin. 2003; 29:1133-1146.10.1177/0146167203254545 [PubMed: 15189609]

Freeston MH, Pléchaty M. Reconsiderations of the Locke-Wallace Marital Adjustment Test: Is it still relevant for the 1990s? Psychological Reports. 1997; 81:419-434.10.2466/PR0.81.6.419-434 [PubMed: 9354091]

Gartstein MA, Rothbart MK. Studying infant temperament via the revised infant behavior questionnaire. Infant Behavior \& Development. 2003; 26:64- 86.10.1016/S0163-6383(02)00169-8

Gotlib IH, Lewinsohn PM, Seeley JR. Symptoms versus a diagnosis of depression: Differences in psychosocial functioning. Journal of Consulting and Clinical Psychology. 1995; 63:90100.10.1037/0022-006X.63.1.90 [PubMed: 7896995]

Hazan C, Shaver P. Romantic love conceptualized as an attachment process. Journal of Personality and Social Psychology. 1987; 52:511-524.10.1037/0022-3514.52.3.511 [PubMed: 3572722]

Hodnett ED, Fredericks S. Support during pregnancy for women at increased risk of low birthweight babies. Birth. 2003; 30:278.10.1046/j.1523-536X.2003.259_1.x

Houts RM, Barnett-Walker KC, Paley B, Cox MJ. Patterns of couple interaction during the transition to parenthood. Personal Relationships. 2008; 15:103-122.10.1111/j.1475-6811.2007.00187.x

$\mathrm{Hu}$ LT, Bentler PM. Cutoff criteria for fit indexes in covariance structure analysis: Conventional criteria versus new alternatives. Structural Equation Modeling. 1999; 6:155.10.1080/10705519909540118

Little TD, Cunningham WA, Shahar G, Widaman KF. To parcel or not to parcel: Exploring the question, weighing the merits. Structural Equation Modeling. 2002; 9:151-173.10.1207/ S15328007SEM0902_1

Locke HJ, Wallace PM. Locke-Wallace Marital Adjustment Scale and prediction test: Their reliability and validity. Marriage and Family Living. 1959; 21:251-255.10.2307/348022

Logsdon MC, Usui W. Psychosocial predictors of postpartum depression in diverse groups of women. Western Journal of Nursing Research. 2001; 23:563-574.10.1177/01939450122045384 [PubMed: 11569330]

Martin RP, Noyes J, Wisenbaker J, Huttunen MO. Prediction of early childhood negative emotionality and inhibition from maternal distress during pregnancy. Merrill-Palmer Quarterly. 1999; 45:370_ 391.

Matthey S, Ross-Hamid C. The validity of DSM symptoms for depression and anxiety disorders during pregnancy. Journal of Affective Disorders. 2011; 133:546-552.10.1016/j.jad.2011.05.004 [PubMed: 21641046]

Norbeck JS, DeJoseph JF, Smith RT. A randomized trial of an empirically-derived social support intervention to prevent low birthweight among African American women. Social Science \& Medicine. 1996; 43:947-954.10.1016/0277-9536(96)00003-2 [PubMed: 8888464]

O'Donnell K, O'Connor TG, Glover V. Prenatal stress and neurodevelopment of the child: Focus on the HPA axis and role of the placenta. Developmental Neuroscience. 2009; 31:285292.10.1159/000216539 [PubMed: 19546565]

O'Hara M, Swain A. Rates and risk of postpartum depression: A meta-analysis. International Review of Psychiatry. 1996; 8:37-54.10.3109/09540269609037816

Olds DL, Eckenrode J, Henderson CR, Kitzman H, Powers J, Cole R, Luckey D. Long-term effects of home visitation on maternal life course and child abuse and neglect: Fifteen-year follow-up of a randomized trial. Journal of the American Medical Association. 1997; 278:637-643.10.1001/ jama.1997.03550080047038 [PubMed: 9272895]

Pasch LA, Bradbury TN. Social support, conflict, and the development of marital dysfunction. Journal of Consulting and Clinical Psychology. 1998; 66:219-230.10.1037/0022-006X.66.2.219 [PubMed: 9583325]

Pauli-Pott U, Mertesacker B, Bade U, Haverkock A, Beckmann D. Parental perceptions and infant temperament development. Infant Behavior and Development. 2003; 26:27- 48.10.1016/ S0163-6383(02)00167-4

Pietromonaco PR, Uchino B, Dunkel Schetter C. Close relationship processes and health: Implications of attachment theory for health and disease. Health Psychology. in press. 
Rini C, Dunkel Schetter C, Hobel CJ, Glynn LM, Sandman CA. Effective social support: Antecedents and consequences of partner support during pregnancy. Personal Relationships. 2006; 13:207.10.1111/j.1475-6811.2006.00114.x

Rini C, Redd WH, Austin J, Mosher CE, Meschian YM, Isola L, DuHamel KN. Effectiveness of partner social support predicts enduring psychological distress after hematopoietic stem cell transplantation. Journal of Consulting and Clinical Psychology. 2011; 79:64- 67.10.1037/ a0022199 [PubMed: 21261435]

Robertson E, Grace S, Wallington T, Stewart DE. Antenatal risk factors for postpartum depression: A synthesis of recent literature. General Hospital Psychiatry. 2004; 26:289-295.10.1016/ j.genhosppsych.2004.02.006 [PubMed: 15234824]

Ross LE, McLean LM. Anxiety disorders during pregnancy and the postpartum period: A systematic review. Journal of Clinical Psychiatry. 2006; 67:1285-1298.10.4088/JCP.v67n0818 [PubMed: 16965210]

Rothbart MK. Measurement of temperament in infancy. Child Development. 1981; 52:596578.10.2307/1129176 [PubMed: 7249824]

Rothbart MK, Ahadi SA, Evans DE. Temperament and personality: Origins and outcomes. Journal of Personality and Social Psychology. 2000; 78:122-135.10.1037/0022-3514.78.1.122 [PubMed: 10653510]

Rothbart MK, Hwang J. Measuring infant temperament. Infant Behavior and Development. 2002; 25:113-116.10.1016/S0163-6383(02)00109-1

Santor DA, Coyne JC. Shortening the CES-D to improve its ability to detect cases of depression. Psychological Assessment. 1997; 9:233-243.10.1037/1040-3590.9.3.233

Satorra A, Bentler PM. A scaled difference chi-square test statistic for moment structure analysis. Psychometrika. 2001; 99:507-514.10.1007/BF02296192

Spielberger, CD. Manual for the State-Trait Anxiety Inventory (STAI). Palo Alto, CA: Consulting Psychologists Press; 1983.

Van den Bergh BRH, Mulder EJH, Mennes M, Glover V. Antenatal maternal anxiety and stress and the neurobehavioural development of the fetus and child: Links and possible mechanisms: A review. Neuroscience \& Biobehavioral Reviews. 2005; 29:237-258.10.1016/j.neubiorev. 2004.10.007 [PubMed: 15811496]

Vaux A, Burda P, Stewart DE. Orientation towards utilization of support resources. Journal of Community Psychology. 1986; 14:159-170.10.1002/1520-6629(198604)14:2<159::AIDJCOP2290140207>3.0.CO;2-H

Wallace JL, Vaux A. Social support network orientation: The role of adult attachment style. Journal of Social and Clinical Psychology. 1993; 12:354-365.10.1521/jscp.1993.12.3.354

Whisman MA, Davila J, Goodman SH. Relationship adjustment, depression, and anxiety during pregnancy and the postpartum period. Journal of Family Psychology. 2011; 25:375-383.10.1037/ a0023790 [PubMed: 21553959]

Widaman KF, Thompson JS. On specifying the null model for incremental fit indices in structural equation modeling. Psychological Methods. 2003; 8:16-37.10.1037/1082-989X.8.1.16 [PubMed: 12741671]

Yonkers KA, Ramin SM, Rush AJ, Navarrete CA, Carmody T, March D, Leveno KJ. Onset and persistence of postpartum depression in an inner-city maternal health clinic system. American Journal of Psychiatry. 2001; 158:1856.10.1176/appi.ajp.158.11.1856 [PubMed: 11691692] 


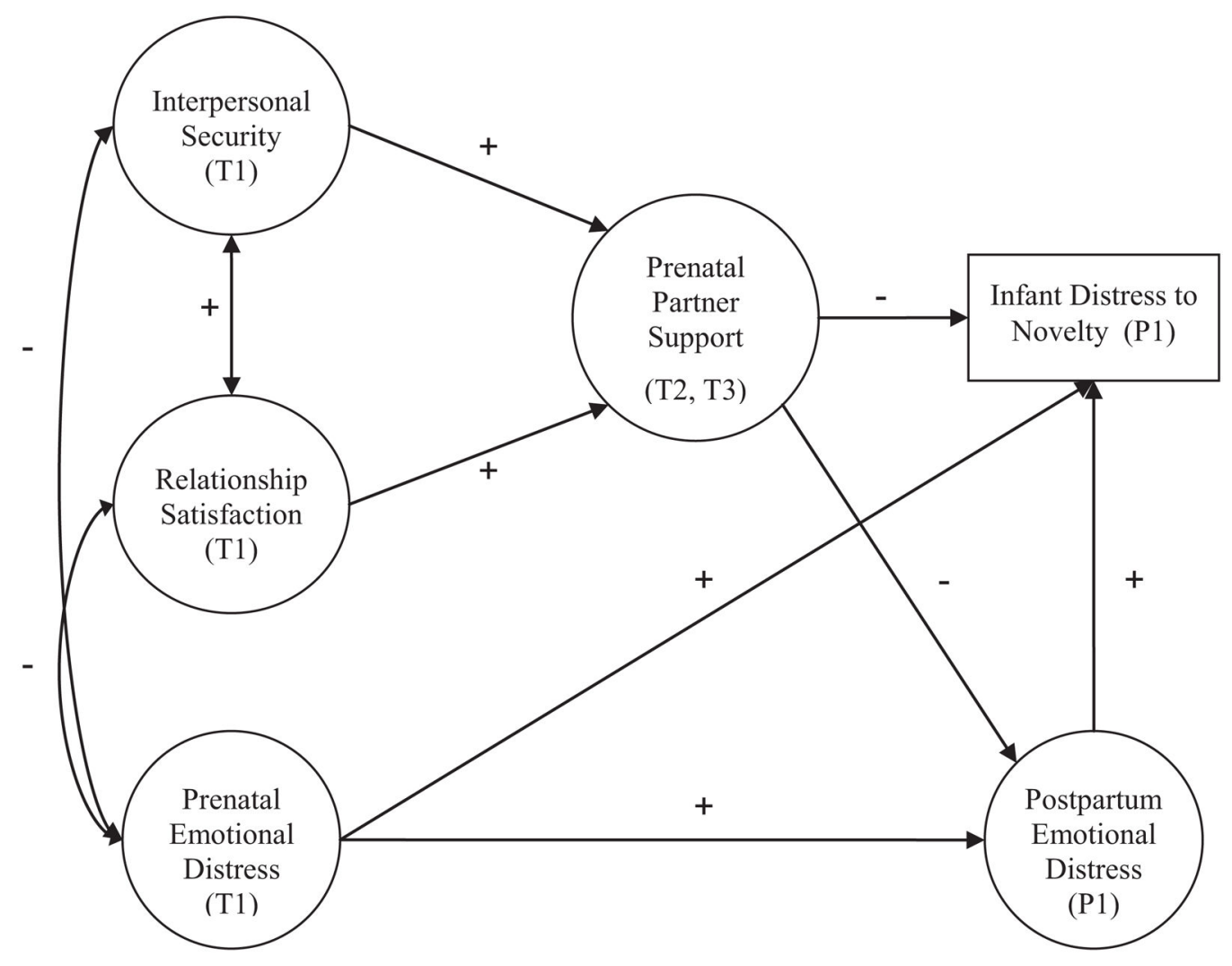

Figure 1.

Hypothesized path model. 


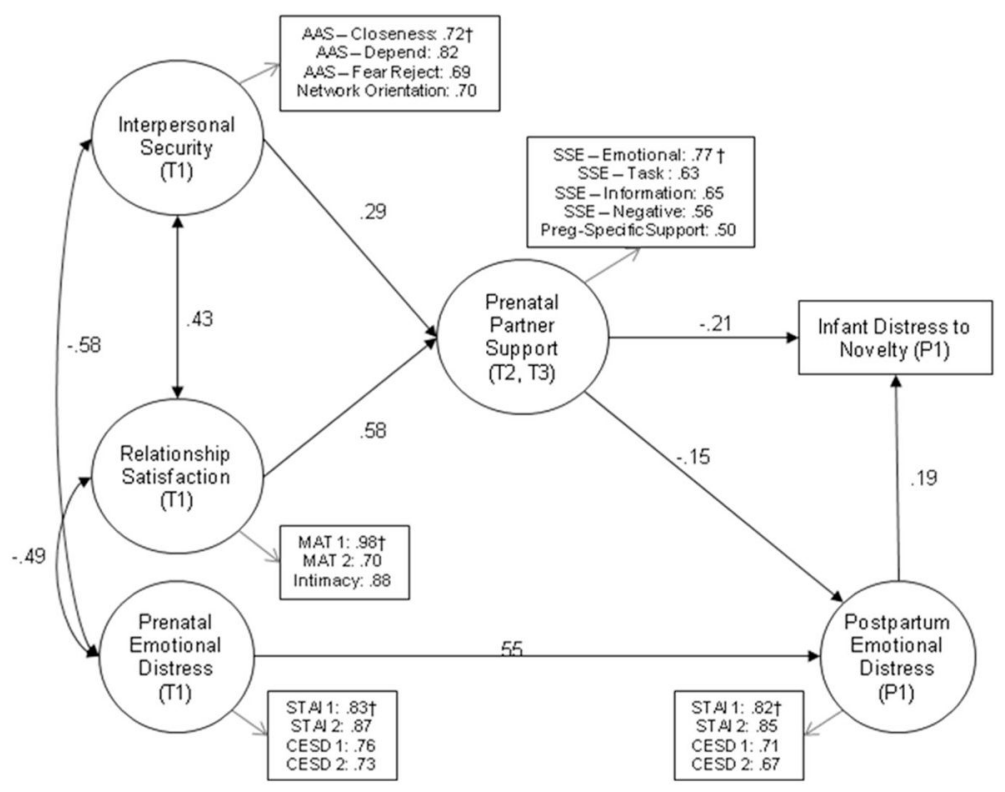

Figure 2.

Final model predicting postpartum maternal emotional distress and infant distress to novelty from prenatal partner relationship variables. Circles denote latent variables; rectangles denote measured variables. One-headed arrows represent regression paths;

two-headed arrows represent correlations. Estimated errors are omitted for visual clarity. Standardized coefficients are presented, and all paths and factor loadings are significant at $p<.02$ (two-tailed). Loadings notated by $\dagger$ were fixed to identify latent factors. Fit statistics: S-B $\chi^{2}(177, N=272)=230.99, p=.004$, robust comparative fit index $(\mathrm{RCFI})=.098$, root-meansquare error of approximation $(\mathrm{RMSEA})=0.034$. 
Table 1

Means, SDs, and Scale Reliabilities for Observed Variables Grouped by Latent Factors

\begin{tabular}{|c|c|c|}
\hline Variable & Mean ( $95 \%$ confidence interval) & Alpha \\
\hline \multicolumn{3}{|l|}{ Interpersonal security } \\
\hline Comfort with closeness (1-5) & $4.12(4.02-4.21)$ & .78 \\
\hline Depend on others (1-5) & $3.47(3.37-3.57)$ & .78 \\
\hline Fear of rejection (reversed; $1-5$ ) & $4.11(4.00-4.22)$ & .89 \\
\hline Network orientation $(1-4)$ & $2.90(2.86-2.95)$ & .82 \\
\hline \multicolumn{3}{|l|}{ Relationship satisfaction } \\
\hline Marital Adjustment Test $(0-158)$ & $124.65(121.82-127.48)$ & .76 \\
\hline Relationship intimacy and closeness (1-7) & $5.85(5.73-5.97)$ & NA \\
\hline \multicolumn{3}{|l|}{ Prenatal partner support } \\
\hline SSE Task (mean of 5 standardized items) & $0.03(-0.05-0.11)$ & .80 \\
\hline SSE Information (mean of 3 standardized items) & $0.01(-0.08-0.10)$ & .63 \\
\hline SSE Emotional (mean of 5 standardized items) & $0.01(-0.09-0.10)$ & .87 \\
\hline SSE Negative aspects (reverse-scored; $1-5$ ) & $3.71(-0.11-0.06)$ & .81 \\
\hline Pregnancy-specific support (mean of 4 standardized items) & $0.00(-0.08-0.08)$ & .76 \\
\hline \multicolumn{3}{|l|}{ Prenatal maternal emotional distress (T1) } \\
\hline State-Trait Anxiety Scale-State Anxiety (1-4) & $1.90(1.82-1.95)$ & .93 \\
\hline Center for Epidemiological Studies-Depression (0-3) & $0.61(.55-.68)$ & .83 \\
\hline \multicolumn{3}{|l|}{ Postpartum maternal emotional distress (P1) } \\
\hline State-Trait Anxiety Scale-State Anxiety (1-4) & $1.78(1.71-1.84)$ & .88 \\
\hline Center for Epidemiological Studies-Depression (0-3) & $0.55(0.48-0.62)$ & .87 \\
\hline Infant distress to novelty (1-5) & $2.04(1.98-2.10)$ & .72 \\
\hline
\end{tabular}

Note. $\mathrm{NA}=$ not applicable; $\mathrm{SSE}=$ social support effectiveness. Response scales for each measure are given in parentheses. 


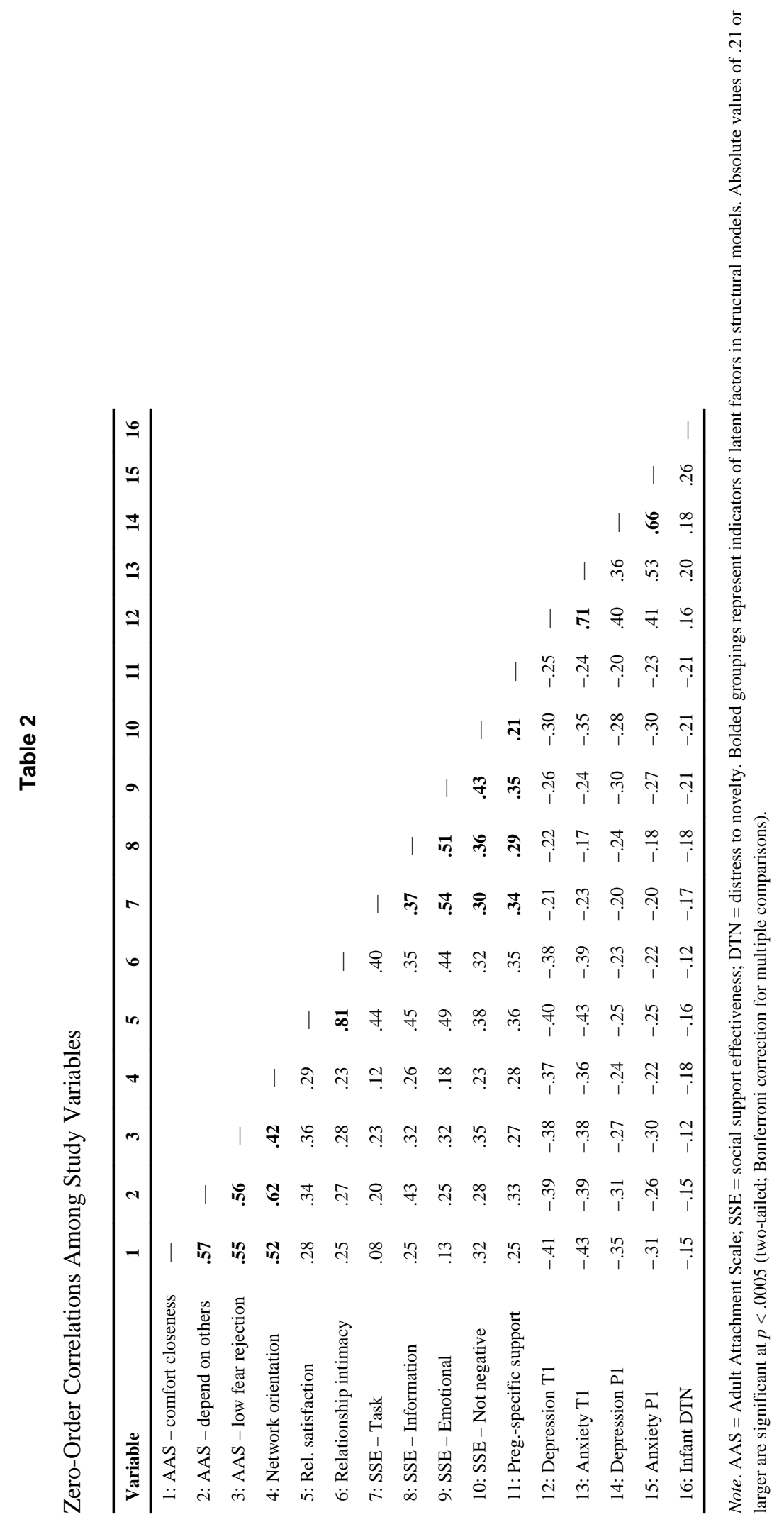

J Fam Psychol. Author manuscript; available in PMC 2014 April 21. 